\title{
GLOBALISIERUNG UND DER KRISE IM RECHT: RETHINKING ETHIK UND MORAL IN DER POSTMODERN ZUSTAND
}

DENDASCK, Carla ${ }^{1}$

SILVA, Adonijah Osias ${ }^{2}$

DENDASCK, Carla; SILVA, Adonijah Osias-Globalisierung und der Krise im Recht: Ethik und Moral in der Post-modernen Staat - fachübergreifenden wissenschaftlichen Erkenntnissen Core Magazin Vol. 1 zu überdenken. Jahr. 1. März. 2016, pp: 98-119-ISSN: 0959-2448

\section{ZUSAMMENFASSUNG}

Dieser Test soll eine kritische Analyse der technologischen und wirtschaftlichen Globalisierung und ihre Auswirkungen auf positives Recht mit ihrer formalen Gesicht Normativista Logik der Kontexte, die zunehmend komplex und veränderlich sind. Ausgehend von dieser Prämisse, es soll beurteilen, wie die Ethik und Moral aus dem Norden in dieser Paradigmenwechsel dienen kann, die notwendig ist für eine Anwendung des Gesetzes, die am besten erfüllt die Wünsche der Bürger in einer Gesellschaft im stetigen Wandel. Ziel ist es, die Rolle des Rechts unter dieser neuen Globalisierung-Bias und seine Bedeutung für den modernen Menschen zu analysieren.

Schlüsselwörter: Ethik. Moral. Die Globalisierung. Rechts

\footnotetext{
1 Promotion in der Psychoanalyse, Postdoc in der klinischen Psychoanalyse, Meister in der Bioethik-Direktor des Zentrums für Forschung und erweiterte Studien-e-Mail: prof.dra.csd@hotmail.com.

2 Rechtsanwalt, Nachdiplomstudium im Steuerrecht; Magister der Rechtswissenschaften an der Escola Paulista de Direito; adonias.Silva@unifesp.br.
} 


\section{EINFÜHRUNG}

General Sabença, die die Flugbahn des Mannes auf dem Planeten, beginnend in den Höhlen bis zum Homo Sapiens, als Man begann Ihre Intelligenz bewusst werden, und legen Sie seine Freiheit die Hindernisse zu überwinden, die immer seinen Weg gekreuzt immer hat ist gekennzeichnet durch die Teilnahme an den Bau von Recht und Gerechtigkeit.

Auch in den Anfängen der Zivilisation, wann hatte eine Vorstellung von verbesserten Recht und Gerechtigkeit als wir haben heutzutage nicht, Man hat immer gekämpft werden, dass der Schauspieler, der seine eigene Geschichte und innerhalb dieser Melodie eine politische und moralische, wurde die agierte in seinem Konzept von Ethik und Moral.

In der Tat kann man unterscheiden, den Mann aus anderen Lebewesen genau für seine intellektuelle Entwicklung, die wiederum direkt mit seinem Verhalten in der Gesellschaft zusammenhängt, $d$. h. in seiner moralischen Verfahren ist nachgewiesen durch ihre Haltung auf kollektiven Zusammenleben mit Gleichaltrigen, die ist nicht der Fall mit dem anderen Lebewesen, die einfach durch Instinkt handeln. Das seine Position unter anderen selbst beweglichen Maschinen der Natur ist verursacht einer herausragende Stellung als soziale Dominator (REALE, 1994).

Seit den gesellschaftlichen Gepflogenheiten vom Vater auf den Sohn übertragen haben die Beziehungen zwischen sozialen Gruppen entwickelte sich aus einfachen Paradigmen der Koexistenz bis die Kommandostruktur solidere wie das PositivadoGesetz und, als Folge der eminent Herdentrieb des Menschen aus ihren sozialen Koexistenz Exsurgir das Recht als gesellschaftliches Phänomen ihrer eigenen Kultur Tat mit seinen Regeln und Sanktionen.

Und, diese Realität zu ratifizieren, es ist ein lateinischer Grundsatz, der sagt: "Ubi Societas, Ibi lus est" (wo es Gesellschaft, es werden ein Recht). Dies ist der Ausgangspunkt dieses Tests, da die Gesellschaft hat sich entwickelt, global geworden ist und positive Recht mit seiner formalen Regelungen, so scheint es, kann nicht mehr 
erfüllen die Erwartungen des modernen Mannes, die in einer globalisierten Welt in seiner ewigen Reise auf der Suche nach Gerechtigkeit folgt.

Globalisierung, die einher mit der technologischen Revolution und die wirtschaftlichen Veränderungen kam, gewann seine erste Phase der Installation, die die Integration der Weltmärkte, jetzt stellt uns vor eine neue Herausforderung sind die Verzweigungen und Auswirkungen auf Rechtsinstitute. Die große Herausforderung ist zu wissen, welche die Wirksamkeit und den Umfang der Rechtsinstitute und Rechtsinstrumente für diese neue Realität des globalisierten Menschen.

Außer, dass du die Rolle der Ethik und Moral des modernen Gesetzes Grundlagen für eine gerechte Gesellschaft und korrekte nicht vergessen kannst. Eine solche Tatsache ersichtlich durch die einfache Tatsache, dass in der Praxis die praktische Anwendung des Gesetzes über verteilte Gerechtigkeit für einen Mann - der Richter - erfolgt, die das Rechtssystem mit der Honourable Mister zur Lösung der Streitigkeiten zwischen den Parteien im Streit auf der Suche nach einem Satz liegt.

Der verfahrenstechnischen Prozess auf der Suche nach dem Satz zu vergleichen, was Sie erkennen, dass werden sie platziert in Konfrontation nicht nur rechtliche, sondern alle ethischen und moralischen Grundsätzen, dass Verwaltungsprozessrecht erfordert nicht nur die Prozessparteien sowie seine eigene Richter. Der Knackpunkt ist wie sein ethisches degenerierte Korruption innerhalb der Globalisierung und ihrer technologischen Systemen erhoben, wo können Sie aus einer einfachen Wahl des Verwalters der Eigentumswohnung bis zu einer Wahl wählen unsere Herrscher unterschlagen.

Wirtschaftliche Globalisierung auferlegt die Pflicht des Mannes Rico, sein zu müssen, um jeden Preis besitzen, und technologische Innovationen sind die Spuren, die die Lokomotive der Unehrlichkeit führt, unter dem Deckmantel der herstellen, der Zweck heiligt die Mittel, weiter und Menschen von ethischen und moralischen Werte aus der Wiege. Und in diesem System die weitergesetzt "Gerson's", der Leichtigkeit um jeden Preis ist das Recht, mit all seinen Aspekten und Determinanten. 
Es geht um die Krise in der rechten Ecke werden in diesem Text, Berücksichtigung von Aspekten der Globalisierung, das hat der Welt ein globales Dorf und nahm mit inm die Werte der Ethik und Moral übermittelt uns von unseren Gründervätern behandeln. Wir leben heute in immer komplexeren zusammenhängen und veränderlich, clamor, die für ein kontextualisiert Gesetz, die den aktuellen Bedürfnissen des Menschen, die nicht mehr der gleiche Mann, der im Rahmen des letzten Jahrhunderts eingefügt sind.

Ausführungen des Betons ist, dass dem Staat und dem Gesetz, nach dem Vorbild traditioneller wir in der Gesellschaft heute im Einsatz sind nicht geeignet, um gesellschaftliche Entwicklungen von Menschen innerhalb der Gesellschaft erfahren. Heute mehr denn je, muss das Recht, andere Richtungen zu nehmen, wenn du die Bestrebungen dieses Mannes treffen willst. Das Recht hat, auf alternative Möglichkeiten zu machen, d. h. das Gesetz muß ein Recht der Verhandlung, der Runden Tische, Schlichtung und Mediation.

Sicher ist, dass die Wege, die uns gebracht das weit nicht das gleiche sein wird, die uns von nun an nehmen. Und aus dieser Realität zu analysieren gilt, inwieweit die richtige Positivado, mit einer formalen Logik und unflexibel, erfültt der Zufriedenheit die Bestrebungen dieser globalisierten Mann, insbesondere hinsichtlich der ethischen und moralischen Aspekt. Ist keine Notwendigkeit, die Ethik und Moral zu halten, ohne das Gesetz und seine korrekte Anwendung auf den besonderen Fall aus den Augen zu verlieren.

\section{GLOBALISIERUNG IM MODERNEN STAAT}

Die Geschichte zeigt, dass Man für die Verbesserung ihrer Lebensbedingungen immer gekämpft hat. Menschliche Konglomerate bereits erfolgte in Form der Städte um 3500 v. Chr. und seinem sozialen Kontext entstanden die Krieger mit ihren primitiven Waffen für die Verteidigung der sozialen Gruppe geschmiedet wurden. Daraus entstand die Konstruktoren der seine Mansarddach, der Einsatz von Wasser dominiert, und diejenigen, die mit seiner Weisheit lernte Naturphänomene zu interpretieren und wurde inre Priester und Richter. 
Von jenem primäre Entwicklung der Mensch und Gesellschaft entwickelte sich in mehrfacher Hinsicht und in mehreren Stadien, dass Sie nicht, analysiert sie, hier seit diesem Papier möchten nicht eignet sich, um diesem Desiderat. Es ist jedoch erwähnenswert hier nur die Abolutismos, die die frühen Tagen des modernen Staates, wird ein Merkmal, das Monopol der Gewaltanwendung durch die Despoten gekennzeichnet, daß absolute Weg die Energie gehalten um jeden Preis, wird nicht nur die Gesetzgeber, sondern auch ihre eigenen Richter, einbrachte.

Der Staat, um die Erhaltung des sozialen Friedens bedroht oder verletzt, Verbot der Autotutela und fordert Verantwortung Streitigkeiten beizulegen. Ab diesem Zeitpunkt hat der Staat nun die Funktion der Anwendung des Gesetzes auf bestimmten Fällen eingereicht und Lösung des Konflikts.

Der Staat als Inhaber der Ordnung, Recht und Gerechtigkeit nur das Feld der Herrscher auf der strengen Grenzen des Gesetzes, geben der Justiz die ausschließliche Zuständigkeit Beurteilungskriterien und Beilegung von Konflikten, und dies macht es daher durch die Zuständigkeit. Die Wort Gerichtsbarkeit stammt aus der Zusammensetzung aus zwei Wörtern: das lateinische Substantiv Jus, Juris, was bedeutet, "rechts", mehr die dritte Konjugation für das Verb Dicere, was bedeutet, "sagen", "sagen", "express". So ist Gerichtsstand der macht, die der Staat hat das Recht zu sagen.

Jedoch die Zuständigkeit ist inert, entsprechend den lateinischen Grundsatz: "Nemo ludex $t$ und Sinus; NE Procedat ludex $t$ Officio (kein Richter ohne Schauspieler, der Richter wird Amts). Damit die Kunst. 2 des Code des Zivilprozessrechts, die so Prolata: "kein Richter bieten den Rechtsschutz sonst Teil oder interessiert zu gelten, in den Fällen und rechtlichen Möglichkeiten".

Aus diesem Grund damit der Zustand die Leistung bieten und Ausübung seine Rolle als Vermittler von Streitigkeiten nicht notwendig, die der Inhaber ist des kollidierenden in das Rechtsverhältnis des Gesetzes Material Interessen kann hat Initiative und gehen Sie klopfen an die Türen der Justiz. Durch geeignete, gilt zu beachten, dass obwohl der Staat nicht gestartet werden, zuständig ist einmal verursacht die rechtliche 
Funktion des Staates, der Prozess von Impuls-Beamten bis zu seiner Fertigstellung mit einem Satz entwickelt werden, die das Geschäft zu beenden.

Mit dem Bevölkerungswachstum und die wirtschaftlichen, technologischen und sozialen Wachstums die Forderung der Bürger, die klopfen an die Türen der Justiz sind proportional größer als die Antworten auf alle, die eine Antwort auf ihre Ansprüche und Streitigkeiten. Und die Folge davon ist, dass die Justiz, die entspricht nicht mehr die Anforderungen dort getroffen, einen gerichtlichen Aktivismus, die nicht die Sorgen der Bürger entspricht.

Heute mit der Globalisierung, die Probleme der Gesellschaft unterscheiden sich völlig von denen erfahren durch eine Generation vor, der Trend ist eine Steigerung dieser Schwierigkeiten und die Gewissheit, die wir haben ist, dass die Justiz für die Änderungen nicht bereit ist, die sich an die nächste Generation zu präsentieren, die wir im folgenden erläutern werde.

Wenn es darum geht, was die Globalisierung im Sinn hat, ist eine seriöse Analyse der Prozesse und miteinander verbunden sind und Veränderungen, die geschehen auf der Weltbühne, und mit ihm der gesamte Weg von denken, handeln und Leben in der Gesellschaft verändert sich erheblich. Diese Änderungen betreffen nicht nur die wirtschaftlichen und sozialen Strukturen, die direkt in der aktuellen rechtlichen Produktion und daher in Form von denken und machen das Recht zu stören.

Der erste Punkt, den Sie nicht scheitern können, zu erwähnen ist die Frage der Souveränität des Nationalstaates angesichts des Wachstums der Multinacionalismo und Transnationalismus. Mit dem Aufstieg der multinationalen Konzerne und die Entstehung des supranationalen und transnationale Agenten grassierende Globalisierung brachte sieht der Nationalstaat seine Stärke im Hinblick auf Verordnung und Strukturierung von wirtschaftlichen Regelungen, als auch in Bezug auf den Schutz des einzelnen und der Gesellschaft gemindert. Was früher die ausschließliche Zuständigkeit des Nationalstaats, aus der Globalisierung das Ziel und Interesse anderer transnationaler und multinationale Institutionen sein wird. 
Multinationale Unternehmen mit ihrer Kapazität für Expansion, sowohl Produktionsund andere Operationen weltweit, trotz seiner Bedeutung für die soziale und wirtschaftliche Entwicklung, auferlegen, das Recht, eine völlig andere Art des Denkens brachte Schimmel im vergangenen Jahrhundert und hat in der Geschichte verewigt worden. Die Leichtigkeit, mit denen diese Firmen haben ihre Fabriken von einem Land ins andere sowie ihre mögliche Welt Handel, verschieben erfordern dem Recht, einen Überblick und offeneren und weniger systematische, geschlossene und liegt als die aktuelle.

Die Relevanz dieser Unternehmen für die Weltwirtschaft führt zu einer Veränderung der Fokus auf soziale Beziehungen, mit all seiner Komplexität erfordern den Rechtsanwalt nicht nur die nationale Perspektive, jedoch einen Blick "über Grenzen", d.h. einen globalen Blick. Sogar sein, dass gemeinsame Stelle wenn Sie hören, dass "die Welt ein globales Dorf ist", was bedeutet, dass die Zäune, Mauern, und die Grenzen, die uns von unseren Nachbarn getrennt kann jetzt nicht mehr flüchtig.

Diese Realität betrifft das Recht auf allen Ebenen und hat seine Entwicklungen in ethische Werte, moralische und gutes Verhalten, die für die Bürger in dieser globalisierten Sozialkontext eingegeben ist. Alle Risiken, die durch Globalisierung, diese Bürger, dass eine gerichtliche Forderung als Antwort auf Ihre Nachfrage dem Recht mehr als Konzepte finden Sie nicht herbeigeführt und Instituten, formell gegründet und bestimmt, wer Ihre Bedürfnisse innerhalb dieser globalen Komplexität nicht erfüllen können.

Aus diesem Grund können wir sagen, dass Globalisierung zeigt auf eine Änderung im Zivil-, Straf-, Verwaltungs-, Geschäfts- und vor allem Völkerrecht, du ständig anzupassen musst, um neue und ungewöhnliche Situationen, die bis vor einiger Zeit galten als vorhanden sind, z. B. den globalen Handel und finanzielle vermarktet Union, die trotz ihrer Flexibilität für Vereinigte Kapital Global erstellen und die Erleichterung der freie Fluss von Investitionen ohne Grenzen zu erfüllen, eine neue Realität und diffuses geltenden Rechtslage. 
Ein weiterer Faktor, der in dieser globalisierten Welt nachdenken dürfen geht das Wachstum der regionale Wirtschaftsblöcke, da sie direkt den Modus Operandi des Schutzrechts ist in unserer Gesellschaft betrifft. Was heute häufiger, und das ist jedes Jahr erhöht hat, ist der Austausch zwischen regionalen Handelsblöcke, nämlich der Abbau von Handelshemmnissen zwischen den Nationen. Denken Sie daran, Abkürzungen, wie nur einige dieser Blöcke zu nennen, die mit kommerziellen Affinitäten beitreten FTAA, MERCOSUR, UEA. Sicher ist, dass der internationale Handel heute eine Realität ist und neoliberale Konzepte wurden mehr dem hegemonialen, öffnen die Türen vieler Nationen früher geschlossen zum Dialog, wie in Osteuropa, der ehemaligen Sowjetunion und mit positiven Reflexionen über China vorkommt.

Den internationalen Freihandel und die Betonung noch verschärft, dass private Märkte irgendwie dezentralisiert und reduziert die Rolle der Regierung, die seit jeher die Regulierungsbehörde für die Wirtschaft. Diese Änderung an den freien Handel mit solche Fahrten, die beeinflusst direkt die Wirtschaftspolitik der "Uncle Sam", als auch die Europäische Wirtschaftspolitik im Allgemeinen zur Gründung von Einrichtungen wie dem IWF, der IBRD (wie auch Regionalbanken und GATT), und ihre Auswirkungen auf die globalisierte Welt verbreitet.

Und wenn diese Änderungen nicht genug um das Diagramm der aktuellen Gesellschaft zu zeichnen, können Sie noch sprechen auf dem weltweiten Trend Demokratisierung, des Schutzes der Menschenrechte, wobei ein viel größeres Interesse an dem Gebiet des Rechts, da die rechtliche Funktion des Staates wies zunächst auf. Parallel dazu die eklatanten Manifestationen von wirtschaftlichen Veränderungen, eine enorme Anstrengung auf internationaler Ebene, für die Erstellung von liberale Politik, die darauf abzielen, zum Schutz der individuellen Menschenrechte sowie die Kontrolle der Schiedsgerichtsbarkeit und Stärkung der rechtlichen Institutionen welche kommen wirklich gerichtliche Anforderung, den Bürgern, gegenüber-ihre diffusen und kollektiven Interessen.

Parallel zu dieser Sie finden auch die NGOs, die transnationalen und supranationalen Akteuren, die in diesem Szenario, zum Schutz der Menschenrechte und die Rechte 
der unterprivilegierten, das Recht auf eine nachhaltige Umwelt, die Rechte der indigenen Völker, die Rechte ethnischer Minderheiten, die Frage der affektiven in Gesellschaft, Homo angezeigt, um nur einige davon zu nennen. Sie dürfen nicht vergessen, dass alle diese Fragen Teil des täglichen Lebens der Bürger sind und Gesellschaft sich auf eine befriedigende Antwort freut und du kommst zu finden, dass die modernen Mann, der, so scheint es, gebeten wurde, die Höhle verlassen, jedoch nicht zu bewegen, bekam deshalb noch alles vertieft sehen Sie um dich herum fühlen sich hilflos und unvorbereitet mit dieser neuen Realität konfrontiert.

Um alle zu analysieren, die dieses globale Problem ist das Patentrecht hat bemühen zu Sanitär und fahren der Konflikte und Auseinandersetzungen entstanden innerhalb der Gesellschaft, weshalb man sprechen sollte, nicht nur in eine radikale und dringend, eine Gesamt-Fitness Konzepte traditionsgemäß benutzt, in begab so, genauso wie der Mann aus der Höhle auch die gleiche Weise zu tun und kommen, dass der moderne Mensch inmitten einer chaotischen Gesellschaft, einer globalisierten Welt und eine Erfahrung des weit verbreiteten Konflikte auf allen Ebenen zu finden.

\section{POSITIVISTISCHEN GESETZ UND FORMALE LOGIK}

Es ist nicht möglich, im Positivismus im juristischen Bereich ohne Schlepptau mit der Figur von Hans Kelsen zu sprechen. In der Tat, obwohl dies den Vater des Positivismus gilt ist hervorzuheben, dass alle ab initio positivistische Lehre im Laufe der Jahre gebaut wurde und seine führende Rolle bei der Entwicklung des Rechts als Wissenschaft hatte.

Für Kelsen sollte das Gesetz als Norma, geschieden von jedem sozialen oder auswertenden Design verstanden werden. Man denke nur an dieses Modus ist, dass das Herzstück seines Schaffens, bekannt als reine Theorie des Gesetzes, die Freisetzung von diesem Rechtswissenschaft auf alle Inhalte, von der Optik des Kelsen, nicht ihr eigenes. Durch den Vorschlag, dass Spalte der deutsche Jurist Rechtsautonomie Wissenschaft für sich soll, ganz frei von Elementen, die nicht Ihnen gehören. 
Für Kelsen sollte juristische Grundlagenforschung unverkennbar, Psychologie, Soziologie, Politik und Moral. Obwohl solche Wissenschaften beziehen sich auf irgendeine Weise nach rechts und Sie sich die meiste Zeit überqueren, kann nicht doch der Rechtswissenschaft mit solchen Konzepten außerhalb seinen Bereich der Sachkenntnis imprägniert. Solches Verständnis, auch wenn es seltsam in den Ohren klingt hat seinen Wert, wenn Sie über Gerechtigkeit, sprechen, als ob eine präzise Sprache verwendet möchten und streng logischen Kelsen richtige Konzept, die Idee der Gerechtigkeit abstrahiert.

Gerechtigkeit ist immer und stets eng verbunden mit den Werten (immer Variablen) angenommenen wer (ob durch soziologische, psychologische und moralische Argumente) aufruft, wird kein Rechtsbegriff allgemein gültig, aufgrund der Ungenauigkeit und Flüssigkeit der Sinne, tun, was ist das richtige für einen bestimmten Kontext oder Gesellschaft, die wäre nicht fair gegenüber anderen.

Jorams Vision das Recht beschränkt sich auf eine Reihe von Regeln durch die Kategorie der Anforderungen dargestellt, "sollte sein", und dabei die Fakten zu einem rechtlichen Maßstab oder Antijurídico bringt. Damit man erkennt deutlich, dass Kelsen eine Ethik basiert auf der Unterscheidung erstellt zwischen sein und sollte. Mit anderen Worten, Kelsen unterscheidet zwischen wie Sachen sind, und wie die Dinge sein sollten und diese Beziehung zwischen "sein" und die "muss-sein" endet in zwei unterschiedliche Aspekte und im Design von entscheidender Bedeutung sind. Die erste dieser Aspekte bezieht sich auf die Tatsache, dass diese Unterscheidung zwischen sein und muss-werden dazu dient, zwischen zwei Arten zu unterscheiden, in denen kann man Jura studieren: die erste ist die Vision des Gesetzes, wie es ist, und die zweite ist die Ansicht des Gesetzes, wie es sein sollte. Der zweite Aspekt, der diese Unterscheidung auch dient dazu, einen umstrittenen Wahrzeichen zwischen dem Reich der Fakten mit Bezug zu werden- und das Königreich der Normen-bezogen werden sollte. Daher die folgende Gleichung: die Nichteinhaltung der Verpflichtung (keine Tatsache gegeben), impliziert Sanktion (sollte sein). In klarere Worte: das Scheitern (Pflicht), es sollte eine Strafe (Sanktion durch den Standard eingerichtet). 
In diesem Zusammenhang Kelsen stellt fest, dass der Standard ein Produkt des Willens, mit dem Ziel ist, menschliches Verhalten Regeln aufgestellt und dient als eine Interpretation. Hier ist es notwendig, um die Positivismus-Standard markieren hat keine Gültigkeit für das Gefühl, fair, aber für die einfache Tatsache zu anderen Standard als überlegen betrachtet er nannte Grundnorm. Es ist von diesem methodischen Konzept der Grundnorm, die der Jurist eine Systematisierung der eine gesamte Rechtsordnung erstellen können.

Einer der seine weitergehende praktische theoretische Konzepte ist die Idee des Rechtssystems als einen gestuften Satz von strukturierten rechtlichen Vorschriften in Form einer Pyramide, abstrakt, punktierte und vorherrsch durch die Staatsverfassung, wodurch die anderen gesetzlichen Regelungen der unteren Hierarchie (die verschiedenen infra-konstitutionelle Gesetze und andere normative Rechtsakte). Diese theoretische Konzeption ist, dass Extrakte das Konzept der konstitutionellen Steifigkeit, die ermöglichen und erfordern ein System zum Schutz der Integrität der Verfassung. Zwar gibt es Kontroversen über die Entstehung der normativen Pyramide, wird für die Zwecke dieses Aufsatzes Kelsen zugeschrieben.

Einen anderen wichtiger Beitrag der Jurist in die praktische Welt des Gesetzes war des österreichischen Verfassung von 1920, verfasst unter seine Inspiration. Im Schatten des Einflusses der Kelseniano dachte, diesen Brief in einem österreichischen Politik, marketing in früheren innovativen positives Recht, das Konzept der konzentrierte Kontrolle der Verfassungsmäßigkeit von Gesetzen und normative als gerichtliche Funktion durch ein Verfassungsgericht fungiert, mit der exklusiven Funktion der Integrität der Verfassung.

Von dort kann die verfassungsmäßige Zuständigkeit in zwei Teile aufgeteilt werden: die verfassungsrechtliche Zuständigkeit (konzentrierte Kontrolle der Verfassungsmäßigkeit) und diffuse verfassungsmäßigen Zuständigkeit (diffuse Kontrolle der Verfassungsmäßigkeit). Dieser letzte Modus der Garde der Verfassung (diffus) wurde in den Vereinigten Staaten von Amerika bereits praktiziert. 
In der brasilianischen Rechtsordnung, unter der Schirmherrschaft der Bundesverfassung von 1988, die verfassungsmäßige Zuständigkeit beide Richtungen praktiziert wird: das Konzentrat durch Aktionen der Kompetenz der Oberste Gerichtshof und die diffuse, ausgeführt in den Aufzeichnungen Aktionen (und Ressourcen, um diese inhärente) in die Zuständigkeit von jedem Gericht, ob Richter und Gerichte.

Daher kann nicht wenn Kelsen diskutieren wir die wichtigste Frage seines Denkens aus den Augen verlieren, die die Gültigkeit der Rechtsstaatlichkeit und deren Hierarchie, dass dies einer der wichtigsten Aspekte seiner Theorie ist.

Bevor die oben ein, dass sehen kann das positivistische-Gesetz ist ein Satz von Regeln menschlichen Verhaltens und diese Normen wiederum, wenn gültig und rechtmäßig empfundene Versagen erwerben Sanktionen wegen seiner Struktur Gründen zeichnet sich als Zwangsmaßnahmen Ordnung und, das gilt nur, wenn Sie eng mit anderen Standard verknüpft sind.

Das große Problem, das sich in Bezug auf Rechtspositivismus präsentiert ist, obwohl er ist ein System von Regeln für das Verhalten des Menschen in der Gesellschaft, aber die Inflexibilität der richtige Positivado kann die Bedenken, dass derselbe Mann nicht erfüllen. Dieses Laufband ist erwähnenswert Kritik Dworkin, Rechtspositivismus sagen, dass Positivismus also dogmatisch ist, dass "das Gesetz oft was sagt der Richter wird" (DWORKIN, 2003, s. 4). Diese Kritik Ronald Dworkin macht einen Kontrapunkt zum positivistischen Gesetz zeigt ein theoretisches Modell, das sich bezieht, um eine adäquate Antwort auf die Frage der Auslegung, die er die Integrität des Gesetzes nennt zu geben

Gut ist das dieses intolerante Dogmatismus des Rechtspositivismus, die letztlich das Gesetz die Anweisung von einigen Richtern reduzieren indem man die Hoffnung der Bürger, Abstiege das Recht nur auf der Welt der Ideen, was sein sollte und nicht in der realen Welt, was erwartet wird, wenn es die Justiz mit einer Forderung eingegeben: die Konkretheit der Klage. Reden Sie nicht über hier in Gerechtigkeit, denn wie bereits erwähnt, der Rechtspositivismus nicht diesen Anspruch vor Gericht, aber einfach zielt 
darauf $a b$, eine allgemeingültige Rechtsbegriff, abgesehen von anderen sozialen Realitäten. Der Punkt hier ist, dass zumindest die so ersehnte rechtliche Bestimmung wirksam in der Tat und im Gesetz und nicht nur Theorie in Rechtsvorschriften, verbalisiert, von Toga.

\section{ETHIK UND MORAL IN DER RECHTEN}

Geht es um Ethik und Moral im Recht, dass man nicht scheitern kann, zu erwähnen, dass das Verhalten eines Individuums gegenüber anderen in jedem sozialen Kontext in gerichtlichen Kämpfe, zu Gerichten durchgeführt, hat immer eine starke moralische Deutung.

Der einzige Unterschied zwischen der ethischen und moralischen Postulate und das Recht ist, dass letztere ihre Zwangsmaßnahmen durch die Rechtsordnung geregelt hat, dass alle überlagert mit inren Regeln und Normen definiert, die die Strafen verantwortlich, die sie einreichen, Diktat mit sich bringen.

Der positivistischen Jurist Hans Kelsen (2001:36), bereits in diesem Text bringt einen wichtigen Beitrag zum Verständnis dieses Thema Ethik und Moral in der rechten Ecke. In seinem Werk "Was ist Gerechtigkeit" wird der deutsche Gelehrte also ausgedrückt:

Erkennen Sie das Recht, als die soziale Ordnung bestimmte Zwangsmaßnahmen Technik, vergleichen wir es mit anderen Ordnungen, in Teil, sie die gleichen Ziele verfolgt, das Gesetz, aber mit unterschiedlichen Mitteln. Das Gesetz ist bestimmten sozialen Medium, kein Selbstzweck. Das Gesetz, Moral und Religion - verbieten alle drei Mord. Aber das Gesetz wird also vorausgesetzt: Wenn ein Mann Mord begeht, ein anderer Mann, benannt nach dem Gesetz gilt gegen die Killer, ein gewisses Maß an Zwang durch die Rechtsordnung vorgeschrieben. Moral erfordern einfach: Du sollst nicht töten.

Es ist bemerkenswert, dass zwar Moral nicht die Kraft und Stärke, eine Sanktion für ihren Ungehorsam herzustellen hat wie das Recht, noch es nicht werden von Gelehrten des Gesetzes seit schon vor die richtige Positivado mit ihrer moralischen 
Standards vernachlässigt kann und Konzepte bereits wurde von primitiven Gesellschaften als Mittel zu leiten und führen die Beziehungen in die soziale Geselligkeit.

Daher die Unterscheidung zwischen dem moralischen und rechtlichen Grundsatz ist eine feine Linie und gehen sie oft zusammen und weiter und die nur durch ihre Auswirkungen getrennt sind. Lassen Sie uns nehmen Sie als Beispiel einen Sohn, dass beim Übergeben von seinem Vater und ein Kompliment erhält nicht die gleiche Höflichkeit zu erwidern oder sogar lenkt, ein solches Verhalten ist eine moralische Handlung, wofür gibt es eine rechtliche Schmerzen. Jedoch wenn diese gleichen Sohn, von seinem Vater begrüßt zu werden einen Personenschaden verursacht, in diesem Fall begeht Verbrechen gemäß Planung und muss bestraft werden.

Aus diesem Grund Chaïm Perelman (1996:289), der große Philosoph von Brüssel und einer der größten Philosophen des 20. Jahrhunderts. XX, um dieses Thema und Sittengesetzes, Links Folgendes eingetragen:

Traditionell, Studien, die die Beziehungen zwischen Recht und Moral gewidmet bestehen, im Geiste Kant'schen in den unterschieden: das Recht regelt das Verhalten; die Moral betont die Absicht, das Gesetz sieht eine Korrelation zwischen den rechten und Pflichten, die moralischen Pflichten vorgeschrieben, die subjektiven Rechte keinen Anlass, das Gesetz regelt Pflichten verankerten macht; die Moral Sanktionen organisierten entweicht. Rechtsanwälte, unzufrieden mit positivistischen Konzeption, Statistiken und Bildgestaltungen, bestehen Sie auf die Bedeutung des moralischen Elements in den Betrieb des Gesetzes, in der Rolle, Gutgläubigkeit, Arglist, Vorsatz, Moral und so viele andere Begriffe, deren ethische Aspekt verachtet werden, kann nicht, spielen.

Durch die Worte des Philosophen erhalten Gelehrter von Brüssel bereits eine Vorstellung über das Ausmaß, die Moral und Ethik in der Anwendung des Gesetzes bis zum heutigen Tag zu spielen. Wir leben in einer Gesellschaft, wo die ethischen und moralischen Werte zu verwechseln mit den persönlichen und privaten Interessen, weshalb diese Werte zunehmend in den Hintergrund, Merkmale einer 
Konsumgesellschaft verbannt werden wo darauf ankommt, was zu erreichen im Sinne, unabhängig vom verwendeten zu tun, dass das alte Prinzip herrscht, dass der Zweck die Mittel heiligt.

Denn es genau in diesem schmalen Grat zwischen der moralischen und ethischen Werte und Moral ist, dass das Recht seinen Weg gehen muss, sobald die Firma kommt, verlieren diese Werte zu jeder Generation. Zur Zeit unserer Väter versiegelt das einfache Wort eine rechtliche und Geschäft wurde bis Umständen weitergeführt.

Derzeit, auch wenn umgeben von allen Garantien, Sicherheiten und alles, aber du hast die echte Gewissheit, die die Rechtsgeschäfte kommen Begriff in einer Weise, ohne das Eingreifen des Staates Ausübung seiner Rolle Coator verlangen die Vorschriften zunächst Compactuadas.

Auf diese soziale Verpflichtung, die vorhanden, rechts auferlegt wird, die Art und Weise materialisiert sich in den Prozess, durch die die Entscheidung der Jury, bringt die gesamte Auflistung der menschlichen Kultur im Interesse der Prozessparteien angesichts das Landgericht, das zunehmend seine Rolle von der Delegat Coator Gesellschaft nimmt.

Sicher ist, dass in vergleichen, die Praxis der Verfahren werden in Konfrontation gebracht, nicht nur rechtliche Regeln, sondern auch alle ethischen Grundsätze, die das Gesetz des Prozesses der Prozessparteien und sogar fordert der Richter selbst. Flankiert von Freiheit, die gibt es eine ganze Struktur der Loyalität und Aufrichtigkeit, die in die sorgen und justizielle Krawatten gesetzlich vorgeschrieben ist. Was Sie bekommen sollten, ist nicht nur die Positivada Standard und Formal, aber auch ethische und moralische Werte als wesentliche Elemente des Anspruchs beantragt gerichtliche Überprüfung.

Die Art und Weise die Gesellschaft heute mit alle Instrumente der sozialen Kontrolle und der Verbesserung der Information über Technik und Wirtschaftswachstum aufgrund von Globalisierung, organisiert ist eignet sich nicht mehr um eine bloße Zuschauer der Gerechtigkeit. Im Gegenteil, Mangel des rechtlichen Rahmens hat 
gezeigt, was heute beobachtet ist mehr denn je ist eine Gesellschaft, die versucht, das Gesetz in die eigenen Hände versuchen, das Vakuum hinterlassen durch die Justiz auf gerichtliche Rechenschaftspflicht zu füllen, aber leider Funktionieren des falschen Weg, machen, oft unter dem Deckmantel der Gerechtigkeit, Ungerechtigkeit noch mehr. All das für diesen juristischen Formalismus, die sucht nur in Technik und Verbesserung der Gesetze, dass Antworten auf soziale Probleme, die es macht deutlich, dass dieses Modell der modernen Gesellschaft nicht mehr unterstützt wird.

Wie es gilt hier die Worte von dem italienischen Juristen Francesco Carnelutti (2004:60) fest, die hat: "Wenn das Gesetz ist ein Instrument der Gerechtigkeit, nicht die Technik oder die Wissenschaft genug, damit umzugehen." Wird belegt durch die Worte von italienischer Jurist-Ínclito, die die praktische Anwendung der Gesetze, die Sie von der Justiz zu tun in rechtliche Konflikte durchgeführt, erfordert mehr als juristische Techniken und Verwaltungsstandards für Gerechtigkeit. Die große Frage, die Sie nicht verstecken können ist, ob die direkte Anwendung wie es gemacht wird, diese Tage, Positivado, bereit und formalen, wirklich Gerechtigkeit visa. Man kann sehen, dass die Anwendung der praktischen Probleme brachte in der Justiz sind nicht vor Prästabilierte Rechtsbegriffe und streng formale Antwort.

Es war nicht ohne Grund, dass Jhering (2012:322) sagte, dass die praktischen Probleme nicht gut anpassen das Paradies der Konzepte in diesem viele Juristen bevorzugen, zu leben. Nach João Maurício Adeodato (2012:317), schlug vor, eine weniger dogmatisch Jhering, als Autor die International ist einer der wichtigsten für die Dogmatik des Privatrechts.

Angesichts der Tatsache, dass man erkennt, dass das Gesetz auf der Suche nach einem Gleichgewicht zwischen der positivistischen Vision Normativista von Hans Kelsen, wo der Richter Entscheidung durch Posta (also positives Recht) geprägt werden muss und die extremere Position gehen muss

vorgeschlagen durch die topische Viehweg, die Entscheidung für die Beziehung mit den behördlichen Texten nicht halten sollte. In diesem Zusammenhang schreibt João Maurício Adeodato (2012:313) mit viel Eigenschaft zum Thema: 
Die topische Viehweg scheint dazu führen, dass eine "offene" übermäßige in Bezug auf die gesetzlichen Text, die nur ein Ausdruck Oberteile unter anderem betrachtet wird. Die Postulat notwendige Verbindung mit dogmatischen Text weigern, topische macht "Orientierung über" eine Methode zu frei und nähert sich der Entscheidungsbefugnis.

Was passiert, wenn versuchen zur Rettung dieser Dogmatismus des positiven rechts ist sucht, nur und nur durch den Staat ihre Unterstützung Basis, zu Lasten der moralischen und ethischen Argumente bei der Suche nach Gerechtigkeit Maßstäbe. Was ist von größerer Bedeutung ist das aktuelle Rechtssystem, Estatalmente Positivado und eigene äußerst formellen Regeln gelten.

Als Folge dieser formalen und starre Landschaft, die nicht mehr erfüllt die Wünsche des modernen Menschen ist, dass die Alternative heißt der Konfliktbeilegung, z. B. Mediation, Schlichtung, Vermittlung, wer sich als Zusätze an die Justiz versucht es darzustellen. Solche Institute bringen in Ihrer Mitte nicht gerade eine Alternative zu der traditionellen Weise zu lösen, sondern eine Suche Konflikte und Rettung durch die moralischen und ethischen Werte, die sich an der Aktion der Schiedsrichter und Mediatoren manifestieren.

Innerhalb des Szenarios dieser neuen Institute, die sich in unserer Gesellschaft ist, die Leuchten müssen, wie ein professioneller Rechtsanwalt Verhalten, testen seine Ethik und moralische Haltung angesichts Schocks, die zunehmend umstrittene Argument angezeigt werden. Auf der anderen Seite hat das Recht des Kunden, die Sponsoren; auf der anderen Seite, als Widerpart, die Reaktion der anderen Partei, die hat auch seinen Patron-Anwalt.

Es ist in diesem Zusammenhang, dass die Anwalt jedoch ihre schwierige Aufgabe des Clients Verteidigung Intellektuellen, ausüben sollte, stehen blieb und im Einklang mit ihren Glauben an das Recht auf Verteidigung und Justiz sucht, ohne vergessen die ethischen und moralischen Werte, die seine Ausbildung zum gegenwärtigen Zeitpunkt, d. h. alle Ihre ethischen und moralischen Erbe geführt, die im Laufe seiner irdischen Karriere gebildet wurde. 


\section{ETHIK UND MORAL IN DER VERWIRKLICHUNG DER GERECHTIGKEIT}

Sobald die Beziehung zwischen Ethik und Moral analysiert, ist Recht gültige Adresse die andere Seite der Medaille, die die Ethik und Moral in der Ausführung der Gerechtigkeit ist. Wenn das Positivado-Gesetz, wie heute, heißt nur den Standard staatlich kontrollierten und streng formalen, suchen, ohne Berücksichtigung der ethischen und moralischen Werte und die Moral von der Gesellschaft etabliert, lohnt eine ernsthafte Reflexion über den Beitrag der Ethik und Moral in der Verwirklichung der Gerechtigkeit.

Es ist bekannt, dass das Objekt moralische ist Ethik. Die Wort Moral stammt aus der lateinischen, Bedeutung "Zoll", dem, wiederum Teil der Wissenschaft, der nun, Moral wichtig, soziale Leben des Mannes kümmert. Ist der Satz von Regeln zur menschlichen Verhaltens als Tugend. So ist die Moral unerlässlich, um den Inhalt des Schutzrechts, vor allem, wenn es darum geht, Durchführung der gerichtlichen Bestimmung, die durch Gerechtigkeit stattfindet.

Die Wissenschaft der Aufgaben wird als Ethik und die Axiológicos-Prinzipien, die sie regulieren, nicht ohne Ethik bezeichnet. Entsprechend der definierten Jurist Miguel Reale: "Ethik ist die Wissenschaft der normativen Standards." Nur der Mensch hält das Erbe, die unterscheidet sich von anderen Lebewesen, wie bereits erwähnt am Anfang dieses Textes.

Gilt hier die Worte von José Renato Nalini (1997:30), bekannt als einer der wichtigsten Theoretiker der Studie der Ethik in Brasilien, die zum Ausdruck:

Ethik ist die Wissenschaft über das moralische Verhalten der Männer in der Gesellschaft. Es ist eine Wissenschaft, weil es Objekt selbst, ihre eigenen Gesetze und proprietäre Methode hat. Das Objekt der moralische Ethik. Die Moral ist einer der Aspekte des menschlichen Verhaltens. Der Ausdruck von der Roman Wort Sitten mit einem Sinn für Manieren abgeleitet ist, wiederholte Satz von Standards, die durch Gewohnheit erworben seiner Praxis. 
Daher kann man annehmen, dass Ethik die Wissenschaft, die die Handlungen des menschlichen Verhaltens im sozialen Kontext auf der Grundlage von Treue und Anstand ist, Glätte der Rechtsakte und würde von der Körperhaltung, so dass die Suche nach Wahrheit ist das größere Ziel erreicht werden, über persönliche und Partikularinteressen der Leidenschaften. Leider, was beobachtet wird ist, dass die persönlichen Interessen, die moralische und ethische Werte und der wirkliche Wunsch der Gerechtigkeit überwunden haben, und es ist diese Situation, die in dieser globalisierten nicht mehr aufrecht erhalten werden kann und Informationsgesellschaft.

Was kann nicht vergessen werden, ist, dass Ethik in diesem aktuellen Voraussetzung um ein rechtschaffenes Leben in dieser globalisierten Gesellschaft führen wird. Ohne Ethik das Leben in der Gesellschaft unerträglich ist und Loyalität, die ihre wichtigsten Tugend ist, sollte nicht nur die Beziehung, aber damit werden Sie den Kern seiner Essenz leiten.

Es ist nicht ohne Grund, dass das Studium der Ethik über Jahrtausende und im Alter kommt und es Anlass zur Sorge für die antike und klassische Gelehrsamkeit, deren Beiträge war bis heute anzusehen wenn es um Ethik und moral Thema geht.

Sie können nur als Beispiel nennen Sie Aristóteles9, wer in jungen Jahren als Schüler Platons zugelassen (der mit 41 Jahren von Philip von Macedon, Erzieher von Alexander die großen ernannt wurde) und unerschöpfliche Quelle der menschlichen Weisheit gewidmet seinen Sohn Nikomachos, geboren von seiner zweiten Ehe Herpile von Stageira, eines seiner Werke für Ethik, die noch heute in allen Teilen der Welt veröffentlicht wird.

Nach Robison Barone (2012:23), in seinem Anwalt Berufsethik Grundierung, zitiert die Estagirista wie folgt: "... Dies ist keine theoretische Studie als andere, weil wir studiert, um nicht zu wissen, was die Tugend, aber gut, zu sein, die sonst nicht zu gebrauchen würde" (Buch II der Nikomachische Ethik). 
Dennoch lehrt Miguel Reale (1994:24), eine weitere wichtige Jurist der nationalen Szene, dass Aristoteles in das achte Buch der Nikomachische Ethik, seine Theorie der Gerechtigkeit entwickelt, und er sagt:

... das Adjektiv unlauterer gilt für zwei Arten von Menschen: 1) für diejenigen, die nicht das Gesetz gehorchen; 2) diejenigen, die mehr als ihren Anteil erhalten möchten, sollten also den Teil, der durch Gesetz sie gewährt werden. Im Gegensatz dazu ist die Messe, was das Gesetz gehorcht und was ist mit seinem Inhalt.

Konnte nicht umhin, aber denken Sie daran die illustre Baruch Spinoza (20 10) oder Bendictus Spinoza, wie er selbst in seinen Schriften, signiert, die auch mit diesem Thema der Ethik, die einen hermetischen Stil zu entwickeln, da die Definition des Gottes, durch die Pfade auf der Suche nach der Freiheit der Seele, die Macht der Vernunft gestört.

Es ist daher durch die Worte dieser edlen Denker, dass Ethik ihre herausragende Rolle bei der Umsetzung des Gesetzes und auf der Suche nach Gerechtigkeit hat. In der Theorie auch distanzierte aus Praxis hat behindert und drücken den Kurs der Justiz in den Jahren. Es lohnt sich wiederholen, was über die Komplexität der Probleme gesprochen worden ist, die in dieser globalisierten Gesellschaft zu erkennen sind.

Sicher ist, dass der Weltgeschichte wird in einer schwindelerregenden Geschwindigkeit geschrieben und beschleunigt, im Gegensatz zu früheren Generationen, wo die Entwicklungen erhielten die langsame Schritte, und hatte die richtigen Bedingungen zu einem gewissen Grad selbst aufrechterhalten und die auf die Sorgen der Bürger angemessen reagieren.

Heute hat der moderne Mensch mit dem Fortschritt der Wissenschaft, die neuen Entdeckungen im genetischen Bereich, Wunder und beängstigend InternetWachstum, Leben in Labors, das Klonen von Menschen, die Langlebigkeit des Lebens, den Fluss von Nachrichten, die Faszination mit dem Computer und der Epidemie von Mobiltelefonen generiert, nicht mehr die gleiche Gewissheit, dass ihre 
Vorfahren. Jetzt alles relativ ist: die Transmuda heute auf gestern in der Geschwindigkeit eines Atoms.

Innerhalb aller Entwicklung und Spitzentechnologie, das einzige, was der Mann nicht, bis jetzt war die Zauberformel der Solidarität, Respekt für den anderen Mann, nicht das Schlimmste und grausamste Art Raubtier. Leider für Ernüchterung dieser Menschheit, die mit modernster Technologie und den Fortschritt der Wissenschaft und große Entdeckungen verzaubert, der Terror wurde die ethische Haltung andererseits fungiert heftig als geistlose Tier, nicht nur ausländische, aber auch Recht reißen, wenn gegen einen wütenden, solche Interessen schützen wollen.

In diesem Zusammenhang ist davon auszugehen, dass Moral und Ethik Schlüsselakteure für die Rettung der Gesellschaft beschädigt und eifrig für Recht und Gerechtigkeit sind. Heute mehr denn je, ist es dringend die Ethik in unserer Gesellschaft zu rehabilitieren, um zurück zu seiner würde den Mann zu bringen. Die Gewalt, Egoismus, stolz und Gleichgültigkeit für seine ähnlich sind, sind die Grundlagen für den Verlust von Moral und Ethik. Es reicht nicht, die würde der menschlichen Person im normativen Akt zu erkennen, wenn ihr das persönliche Verhalten in der Gesellschaft erfahrene geleitet wurde.

Sicher ist, dass Ethik und Moral, einmal eingelöst, werden Triebwerke und Treiber zu einer Zukunft der Hoffnung und Verheißung für die Menschheit, die zwar mit so viel Technologie und Entwicklung, konfrontiert geworden ist, die kleinlichen Praktiken und primär, dass mehr auswärts Männer soziale Geselligkeit, die integrieren. Dies ist die große Herausforderung moderner Gesellschaften, das ist die Rolle der Ethik in der Schwelle des dritten Jahrtausends.

\section{DIE BEDEUTUNG DES RECHTS FÜR DEN MODERNEN MENSCHEN}

Die Relevanz, die das Recht in der modernen Gesellschaft strukturelle Krise spielt behandelt Fulcralmente, verbreitet und bringt zum Ausdruck, dass Descortinamos in unserem Land, welche endet generieren eine Zeichenfolge der Nebenwirkungen, die weit verbreitet sind. Der Mangel an moralischen und ethischen Werte dort beginnen 
von Herrschern, die anstatt Beispiel Glätte und Redlichkeit, lieber gehen den umgekehrten Weg, unter dem Deckmantel der Straflosigkeit, die Revolte den Bürger, der Ihre Steuern zahlt, verankert und kämpfen, um ein menschenwürdiges Leben zu pflegen und zu korrigieren. Für eine weitere Schicht einige Bürger erschüttert durch diese Situation zu beheben, genauso zu handeln, unter der Begründung, dass bei der Fehler kommt von dort oben ist kein Grund nicht auf die gleiche Weise handeln.

Diese anarchische Panorama und Chaos ist, dass Bürger in ihren Tag zu Tag Gesicht und nicht müde, bis wann Fragen weiterhin beobachten alle diesem kraftlos, ohne Aussichten für Änderungen, beteiligt ein Rechtssystem, das mit einem komplexen Satz von steril, Gesetze können nicht solche Bürger erstellen Abwehrmechanismen, die die staatliche Struktur mit seinen schweren Steuern verwaltet kein Beton zurück. Sicher ist, dass die Möglichkeit oder die Aussicht auf eine Änderung in diesem Zustand der Dinge durch ernstes und dringendes Berücksichtigung der die juristische Institute und deren Anwendung in der Gesellschaft, die ließ etwas zu wünschen übrig in der gerichtlichen Bestimmung gehen sollte.

Es gibt eine Menge Material zu diesem Thema geschrieben, und der Trend im Laufe der Zeit ist, dass weitere Juristen und Wissenschaftler des Rechts zurück, Ihre Augen auf dieses Problem im Sinne einer Änderung der Richtung der Richtungen, die das Recht, nicht nur in unserer Gesellschaft genommen hat, aber warum sagen Sie nicht, die Welt als Ganzes, nämlich das Scheitern, die Interessen des Bürgers zu respektieren, die Justiz sucht , zu Lasten der diese zügellose Korruption, der tobt und die Justiz, genau wegen bloßen persönlichen Interessen in Ungnade fallen lassen.

Für Lênio Jurist Streck (2009) füllen nicht das Recht diese Lücke in unserem Rechtssystem, die verewigt, die Politik der Straflosigkeit und des Willens der Justiz nicht zu erfüllen die Erwartungen der Bürger, nicht aus Mangel an Voraussicht in das Rechtssystem, wenn nicht wegen eine "Krise", wie er es nennt. Was schlägt der Jurist Gaucho ein Paradigmenwechsel vom aktuellen rechtlichen Modell Bereitstellung von in unserer Gesellschaft, die sehr weit von der aktuellen Realität in unserem Land ist. 
Es ist genau diese "Krise" von der Rio Grande-Wasser-Teiler-Jurist, die das Verhalten der Anwalt in dieser komplexen und globalisierten Gesellschaft durchführen werden erkannt. Die alten Verbrechen so angewidert von der Moral der primitiven Höhlenbewohner gehen zurück jetzt auf eine noch größere Grausamkeit begangen werden. Hinzu kommt, dass andere Verbrechen unserer Generation, als die elektronischen Verbrechen wie das Klonen von Kreditkarten, Guthaben bei kriminellen Bewegung durch elektronische Waagen, Mendaz e-Mails mit Links, die Passwörter für kriminelle Zwecke, unter anderem zu erfassen, die nur der menschliche Geist ist zu weben und ausführen wollen.

Nun, ist es in diesem Kontext chaotisch und unübersichtlich, dass wir die Relevanz des Rechts für den modernen Menschen gefunden weil das Recht, in ihrem Streben nach Gerechtigkeit, ist das Zentrum der Betrachtung der all diese Probleme, die der moderne Mensch sind. Seit die persönlichen Dramen des Familienrechts mit seiner kalten Gesundheit bis zu den absoluten Unbeweglichkeit der alles, was wir, angesichts der Tötung unschuldiger Opfer in den Händen eines abgeschossenen "Punisher" verrückt und Durst nach Rache tun können, sollte das Recht ernsthaft überdacht werden, um betrachten die sozialen Missstände, die der moderne Mensch in vollständige Ratlosigkeit und Hilflosigkeit angesichts der Tatsachen entstehen diese Präsenz jeden Tag in der Gesellschaft.

Die Relevanz der rechten Seite, wenn Sie auf die moralischen Gesellschaft Involution in den Ablauf der öffentlichen Manager zu tun, die Excel anzeigen machen dem Gesetz ein Instrument sein, gegen die Interessen der regierten unbeholfen und ungeschickt im Umgang mit diesem Problem wird. Das Recht muss die persönlichen und ideologischen Interessen erklingen, und wenn Sie in werfen die Suche nach diesem Szenario Änderungen, die jetzt präsentiert sich.

Das Reich der brute-Force wurde, was in unserer Gesellschaft durchgesetzt hat. Im Umgang mit rechtsverletzenden Rechte die Gerichte von der Waage der Gerechtigkeit nie für den Bürger vor dem Staat, viel weniger zugunsten der Armen gegenüber die reichen mehr zu oszillieren oder sogar vor den privilegierten gegen eine anonyme. 
Nur um eine blasse Vorstellung von was gesprochen wird, schaffen die Vorschriften des Zivilprozessrechts Privilegien zugunsten der öffentliche Einrichtung mit Quad, sich zu verteidigen und in Double, und hatten wir schon erwähnt, dass der Staat selbstRichter, in der Person des Richters, gegen seine eigene Entscheidung berufen kann, jedes Mal, wenn sie gegen den Staat ausgestellt wird.

Und noch schlimmer zu machen, sind die vorgeschlagenen Justizreformen in unserer Gesellschaft nie zu Gunsten der Bürger Litigator; im Gegenteil, alle Reformen in Brasilien hat wandte sich gegen die Prozesspartei in einem vergeblichen Versuch, versuchen, die Gesellschaft zu überzeugen, dass Gerechtigkeit langsam ist und dass die Verzögerung bei der Bereitstellung von gerichtlichen nicht nur der Justiz selbst überprüfen.

Dieser geht zurück auf den gegenwärtigen Moment Leben wir in, das ist eine unersättliche Suche für die Rehabilitation von Ethik und Moral in der Welt, so, dass eine Mindestnorm Verhaltensregeln in Akten des Lebens in der Gesellschaft und im Falle des Rechts, in alles, was die Rechte der Bürger betrifft. Rechtsanwälte, Richter, Staatsanwälte, alle zusammen sollten Bauherren eine neue rechtliche Realität, in der ständigen Suche nach ethischen und moralischen Werte im Streben nach Gerechtigkeit.

Alternative Konflikt Lösung Institute füllen wie bereits erwähnt, Mediation, Schlichtung und Schiedsverfahren, wir freuen uns in diesem Neuerstellung Prozess und die Suche nach Gerechtigkeit, da er erhebt keinen auf Ersatz der Justiz Anspruch, sondern als Nebendarsteller dieser Suche für etwas neues, und dass kommt in der Tat, die ideale der Gerechtigkeit vergessen und im Laufe der Geschichte außer acht gelassen.

\section{FAZIT}

Vor allem, die was gesprochen wurde, das Recht aufzugeben, diese dogmatische und geschlossene vorausgesetzt im Moment braucht und muss gehen Sie in Richtung mehr Plural. In dieser globalisierten Welt mit allem, was die Stränge hingewiesen haben, gibt es keinen Platz mehr für eine starre, einheitlicher Vision und sehr formal, 
im Gegenteil, man kann sprechen jetzt in Vielfalt der gesellschaftlichen Akteure (Rechtsanwälte, Richter, Psychologen, Techniker) und Pluralismus rechtliche Institute, die am besten den Bedenken, dass moderne Gesellschaft, einschließlich Schiedsverfahren, Mediation und Schlichtung entspricht.

Gegenwärtigen Zeitpunkt führt zum Ersatz für die Individualisierung der Rolle des Richters in einem Prozess der gemeinsamen Verantwortung der anderen Akteure im kollektiven Prozess der gesellschaftlichen Veränderungen (die Akteure außerhalb der Justiz, wie die Profis-Retromencionados), alle vereint auf der Suche nach eine solide, spürbar und konkrete Lösung für die Bedürfnisse der aktuellen Mann. Die Justiz muss Förderung und Erleichterung die Produktion von Erfahrungen, die die Verbindung zwischen sich und der Gesellschaft statt Disjunktion zu fördern, der Fragmentierung und der Abgelegenheit des Bürgers von Ihrer Sehnsucht nach Gerechtigkeit.

In diesem Sinne sollte die Justiz selbst Partnerschaften fördern und Harmonisierung der rechtlichen Praktiken mit den Ämtern bereits erwähnt, dass wenn Sie, von Erfahrung wissen, in der Lage, eine mehr angemessene und zufriedenstellende Rendite für den Bürger wurde. Sollte nicht hier gibt es Angst vor der Konkurrenz, viel weniger die Angst vor dem scheinbaren Widersprüche, die kommen können, mitten in diesem Prozess des Wandels entstehen zu lassen. Die Widersprüche müssen gearbeitet und nicht gelöscht, da, obwohl einige von ihnen apriorische, unüberwindbar mag, noch nicht gelähmt bleiben muss oder Leckage und Wissenschaftsgeschichte setzt sich aus Konstruktion und Dekonstruktion von Paradigmen und Überwindung der Gegensätze.

Für jegliche Änderung gibt es daher für die Justiz, die Produktion von Erfahrungen zu öffnen, die die Vielfalt des Wissens und der Rechtspraxis, Förderung der Partnerschaft, sogar zwischen unterschiedlichen Perspektiven und Theorien der gegenwärtigen "Status Quo" zu fördern. Die Suche für den Dialog mit der "sozialen Bewegungen" und anderen Akteuren, die die traditionellen Formen und Burocratizadas der Beteiligung der Bürger an die eigene Geschichte zu dekonstruieren muss vorhanden sein. 
Heute, mehr als je zuvor, es ist dringend zu verstehen, dass diese rechtliche und politische Rolle spielt die Herausforderungen, die präsentieren sich heute auf den modernen Menschen (oder Postmoderne) und erkennen, dass alle Produktion von Werken, die darauf abzielen, Strukturwandel sozialen Auswirkungen oder besser Spruch hat, dass bestimmte Änderungen alle Produktion stammt und mehrere Machtverhältnisse und Kraftfelder.

Die Wanderung auf den Change-Prozess, der kritischen Sinn und transformative Potenzial offen hängt davon ab, die Arbeit der Justiz kritische Selbsteinschätzung über die Bedeutung und die Folgen ihrer Art zu handeln. Es ist notwendig für Rechtspraktiken auf die Interaktion mit sozialen Bedürfnissen, mit den Herausforderungen der Globalisierung basieren, machen immer einen Kontrapunkt mit der aktuellen Situation der Justiz, die verschrottet wird und keine Mindestvoraussetzungen erfüllen die Erwartungen der Bürger, stehen dort überfordert.

Aus diesem Grund hat die Justiz zu erkennen und andere Akteure, die bereit sind, einen Beitrag zur Lösung von Streitigkeiten und Zufriedenheit der Bürger zu legitimieren. Statt die Frage des Konflikts der Gerichtsbarkeit, die Justiz zu handeln, sollten um an sitzen optimieren die Tabelle mit den anderen Fachleuten mit den vitalen Bedürfnissen des Menschen, über die Probleme herausgegriffen und die Unterschiede. Sein ein Riesenschritt in Richtung einer Änderung der Ethik-Politik, Induktivität und Treiber der Reflexion darauf, nicht nur eine Selbstkritik abzielen würde, aber auch eine echte ändern, die versucht, das Recht auf Ihren wahren Weg, die das Streben nach Gerechtigkeit in einer globalisierten Welt ist, ohne zu vergessen, die Ethik und Moral und Pilaster einer echten leitende demokratischen Gesellschaft auf der Grundlage der Rechtsstaatlichkeit.

\section{BIBLIOGRAPHISCHE HINWEISE}

ADEODATUS, João Maurício. Ethik \& Rhetorik. 5. Auflage. São Paulo: Saraiva, 2012. 
, João Maurício. Legitimierung von rechtlich organisierte Prozedur: zur Theorie von Niklas Luhmann. In: Ethik und Rhetorik: zu einer Theorie der juristischen Dogmatik. s. 53-80, São Paulo: Saraiva, 2002.

BARONI, Robison. Grundierung und Berufsethik Rechtsanwalt. 3rd Ed. LTR Editora Ltda:

BOBBIO, Norberto. Rechtspositivismus: Lehren aus der Rechtsprechung. Übersetzt von Márcio Pugliesi, Edson Bini, Carlos e. Rodrigues, São Paulo: Symbol, 1995.

. Allgemeine Theorie der Politik. Daniela Übersetzung Beccaccia Versiani, Rio De Janeiro: Campus, 3. Nachdruck, 2000.

. Theorie des Rechtssystems. 10. Hg. Übersetzung von Maria Celeste Lamm Milch Dos Santos, Brasília: Ed. Universität von Brasilia, 1997.

Francesco CARNELUTTI. Was hat das Recht geboren. Ricardo Rodrigues Übersetzung Bereich. 1st Ed. Campinas: Editora Russel, 2004.

DWORKIN, Ronald. Das Reich des Rechts. Martins Fontes: São Paulo, 2007. S. 4

Faria, José Eduardo, Recht und Globalisierung - Auswirkungen und Perspektiven, São Paulo: Malheiros, 2010, 1. Auflage, 3. Unentschieden.

FERNANDEZ Jr., Einführung in das Studium des Rechts: Technik, Entscheidung, Herrschaft. 3. Hg. São Paulo: Atlas, 2001.

Sohn, Willis Santiago Krieg. Theorie der Rechtswissenschaft. 2nd Ed. Sami: São Paulo, 2009.

HABERMAS, Jürgen. Und Demokratie: zwischen Faktizität und Geltung. 4. Hrsg.: Vol. 1. Übersetzung von Flavius Beno Sienbeneichler, Rio De Janeiro: brasilianischer Zeit, 1997. 3 V.

Rudolf Von JHERING. Der Kampf um das Recht. Martin Claret: São Paulo, 2003. 
KELSEN, Hans. Das Problem der Gerechtigkeit. 2. Hg. Übersetzung von João Baptista Machado, São Paulo: Martins Fontes, 1996.

. Was ist Gerechtigkeit-Gerechtigkeit, Recht und Politik im Spiegel der Wissenschaft. 3rd Ed. São Paulo: Martins Fontes, 2001.

. Allgemeine Theorie von Staat und Recht. 3. Hg. Übersetzung von Luís Carlos Borges, Sao Paulo: Martins Fontes, 1998.

. REINE THEORIE DES GESETZES. João Baptista Machado zu übersetzen. 6 Ed. São Paulo: Martins Fontes, 1999, Seite 1.

NALINI, José Renato. Allgemeine und berufliche Ethik. Rvista der Gerichte: São Paulo, 1997.

Nunes, Pedro. Wörterbuch der juristischen Technik. 13. Ed. Erneuern: Rio De Janeiro, 1998.

Chaim Perelman. Ethik und Recht. Martins Fontes: São Paulo, 1996.

REALE, Miguel. Eine neue Ethik des Richters. Koord. José Renato Nalini. Magazin der Gerichte: São Paulo, 1994.

Baruch SPINOZA. Die Ethik. Trad. Lívio Xavier. Hg. Tecnoprint S.a.-Ediouro: São Paulo.

VALLE, Gabriel. Étiva und Recht. 1st Ed. Synthese-Verlag: São Paulo, 1999.

SETHI, Vincent Paul. Normen der Berufsethik. Juristischen Verlag Ltda: Festung, 1989.

, Springt von Eugenio Raul ZAFFARONI-Stil. Justiz, Krise, Erfolge und Missverständnisse. Trad. Von Juarez Tavares. Hg. Magazin der Gerichte: São Paulo, 1995. 